SHORT REPORT

\title{
Fertility preservation in adolescent males with cancer in the United Kingdom: a survey of practice
}

\author{
A W Glaser, L Phelan, M Crawshaw, S Jagdev, J Hale
}

Arch Dis Child 2004;89:736-737. doi: 10.1136/adc.2003.042036

$\mathrm{F}$ atherhood is an important issue to young men surviving cancer as fertility may be impaired by treatment. ${ }^{1}$ A number of strategies to preserve fertility are available to adolescent males. Wide variation in practice for adolescent male fertility preservation has previously been shown in North America. ${ }^{2}$ No defining data for the UK are available. In the UK, these activities are regulated by the Human Fertilisation and Embryology Authority (HFEA). ${ }^{3}$

\section{PARTICIPANTS, METHODS AND RESULTS}

A national postal survey of all 22 regional paediatric oncology centres (POCs) and all HFEA licensed assisted conception units (ACUs) actively involved in clinical practice in the UK was performed. The aim was to document common practices and areas of variance, and to identify concerns of multidisciplinary healthcare professionals.

Twenty POCs (90\%) responded; all offered access to storage facilities for sperm and/or testicular tissue for adolescents under the age of 18 years. Sixty five ACUs (62\%) responded, of whom 23 currently offered storage facilities for this group. Only six ACUs named by POCs as their referral unit responded, thereby preventing detailed correlation between paired centres (the other 17 centres may have offered gamete storage to adolescents with either non-malignant conditions requiring chemotherapy or those being treated in adult cancer units). Table 1 shows available collection techniques together with the number of centres actually utilising them. All four units storing tissue from epididymal aspiration or testicular biopsy were doing this as part of a clinical programme with no parallel research programme.

Fourteen POCs (70\%) offered fertility preservation to individuals who had begun but not completed sexual development. No centres offered this service prior to the onset of sexual development. Nineteen (75\%) offered it to individuals with prior exposure to chemotherapy.

Fifteen POCs (75\%) and seventeen ACUs (74\%) required individuals to be Gillick competent for sperm banking, while seven POCs (35\%) and 10 ACUs (43\%) required this for testicular biopsy or epididymal aspiration. ${ }^{4}$ Written information regarding fertility preservation specifically for males under 18 years of age with cancer was reported to be given by 11 POCs (55\%) and two respondent ACUs (9\%). Consent was obtained by a wide range of professional groups, including reproductive medicine physicians, paediatric oncologists, andrologists, and nurses.

Psychosocial support is a statutory requirement for ACUs. It was available for patients in 15 ACUs $(65 \%)$ and in 12 (52\%) for their partners or parents. Twelve ACUs (52\%) felt their staff had adequate training, experience, and qualifications to offer this service.

Written guidelines regarding fertility preservation were available in eight POCs (40\%). Eight of the remaining centres (75\%) had discussed but not implemented guidelines over the previous three years. Twenty $(87 \%)$ of the ACUs working in this field indicated they would welcome national guidelines.

\section{COMMENT}

Variation in available services for the fertility preservation of adolescent males exists across the UK. The forthcoming detailed audit of these activities by the United Kingdom Children's Cancer Study Group and recommendations produced by the British Fertility Society will hopefully clarify some of these issues. ${ }^{5}$ However, this study suggests that statutory requirements are not being met by all centres: ${ }^{1}$ counselling should be available to all and consent is required from all individuals, and not proxies, whenever mature gametes are stored. Thus, those under 16 years of age in Scotland and 18 years in England and Wales must be Gillick competent to undergo this procedure, a condition not being met by $25 \%$ of ACUs for semen donation from adolescents. Interestingly, contrary to medical and parental opinion, adolescents have previously been found to welcome the opportunity to address fertility preservation at a time when cancer chemotherapy is being contemplated.

It is recognised that any child with testicular volume of greater than $4 \mathrm{ml}$ (Tanner stage 2 or above) should be considered as potentially harbouring mature gametes. ${ }^{5}$ Further legislative changes have been recommended, by the McLean Review and British Fertility Society, which would

Table 1 Available and utilised strategies for gamete collection

\begin{tabular}{llllll}
\hline & \multicolumn{2}{l}{ Paediatric oncology centres } & & \multicolumn{2}{l}{ Assisted conception units } \\
\cline { 2 - 3 } \cline { 5 - 6 } Technique & Available & Actually utilised & & Available & Actually utilised \\
\hline Ejaculation & $100 \%(20)$ & $85 \%(17)$ & & $95 \%(22)$ & $\mathrm{N} / \mathrm{A}$ \\
Electroejaculation & $35 \%(7)$ & $28 \%(2)$ & & $26 \%(6)$ & $\mathrm{N} / \mathrm{A}$ \\
Epididymal aspiration & $30 \%(6)$ & $33 \%(2)$ & & $69 \%(16)$ & $25 \%(4)$ \\
Testicular biopsy & $30 \%(6)$ & $50 \%(3)$ & & $69 \%(16)$ & $25 \%(4)$ \\
\hline
\end{tabular}

Numbers of centres or units are in parentheses.

Percentages for "actually utilised" represent percent of centres who have performed the technique for the study population when it is locally available.

N/A, information not available from questionnaires. 
enable mature gametes to be obtained from, and stored for, "non-Gillick competent" gamete providers as long as it is in their best interests. ${ }^{7}$ However, results of this survey indicate that while the consent provisions of the Human Fertilisation and Embryology Act 1990 remain, an urgent programme of education for health care professionals is required.

\section{ACKNOWLEDGEMENTS}

The authors acknowledge the support and advice of the Project Advisory Group. Dr Gill Levitt (UKCCSG Late-effects Group Chair) and Dr Hamish Wallace reviewed the draft manuscript.

\section{Authors' affiliations}

A W Glaser, L Phelan, S Jagdev, Yorkshire Regional Centre for Paediatric Oncology and Haematology, Leeds Teaching Hospitals NHS Trust, Leeds, UK

M Crawshaw, Department of Social Policy and Social Work, University of York, UK

J Hale, Royal Victoria Infirmary, Newcastle-upon-Tyne, UK

Funding: NHS Executive (Northern and Yorkshire) RRCC240SG
Correspondence to: $\operatorname{Dr}$ A Glaser, Consultant Paediatric and Adolescent Oncologist, Children's Day Hospital, St James's University Hospital, Beckett Street, Leeds LS9 7TF, UK; adam.glaser@leedsth.nhs.uk

Accepted 5 January 2004

\section{REFERENCES}

1 Schlover LR, Brey K, Lichtin A, et al. Knowledge and experience regarding cancer, infertility, and sperm banking in younger male survivors. J Clin Oncol 2002;20:1880-9.

2 Glaser AW, Wilkey O, Greenberg M. Sperm and ova conservation: existing standards of practice in North America. Med Pediatr Oncol 2000;35:1 14-18. 3 Human Fertilisation and Embryology Act, 1990. London: HMSO, 1990.

4 Gillick $\vee$ Norfolk and Wisbech Area Health Authority (1985). All England Reports 402, HL.

5 Multidisciplinary Working Group convened by the British Fertility Society. A strategy for fertility services for survivors of childhood cancer. Human Fertility 2003:6(2):A1-40

6 Crawshaw M, Glaser A, Hale J, et al. A study of the decision making process surrounding sperm storage for adolescent minors within paediatric oncology. Research report ISBN I-872159-35-4., University of York, 2003.

7 Mclean SAM. Review of the common law provisions relating to the removal of gametes and of the consent provisions of the Human Fertilisation and Embryology Act 1990. London: Department of Health, 1998.

\section{IMAGES IN PAEDIATRICS}

\section{Radionuclide bone scintigraphy in Engelmann-Camurati disease}

A 3 year old male presented with inability to run, waddling gait, and progressive lethargy for one year. There was bowing of lower limbs, proximal muscle wasting, and preserved deep tendon reflexes and sensations.

Serum calcium, phosphorus, alkaline phosphate, and creatinine phosphokinase were normal, with moderate anaemia and raised ESR. On skeletal survey, cortical thickening and sclerosis of diaphyses of long bones and skull bones (fig 1), diagnostic of EngelmannCamurati disease, was seen. Tc99m MDP bone scan (fig 2) revealed symmetrically increased osteoblastic activity in diaphyseal areas of humeri, femori, tibia, forearm bones, phalanges, and skull bones.

D Narang, B Bharati Department of Paediatrics, Pgimer Chandigarh, 160012, India

A Bhattacharya, B R Mittal Department of Nuclear Medicine, Pgimer, Chandigarh, 160012, India

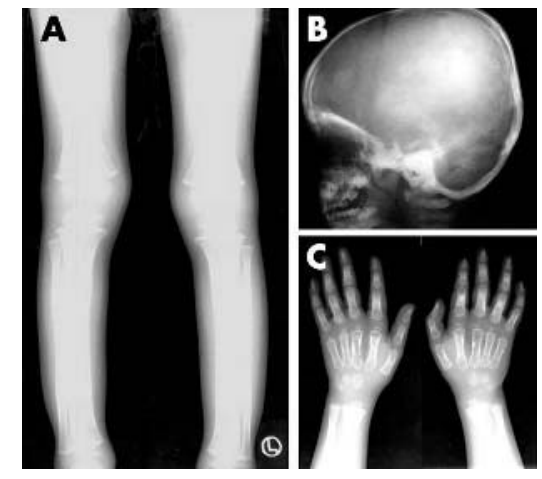

Figure 1 Radiographs of lower limbs $(\mathrm{A})$, skull (B), and hands (C) showing osteoscleortic lesions.

Correspondence to: Dr A Bhattacharya, Assistant Professor, Department of Nuclear Medicine, Pgimer, Chandigarh, 160012, India; shalmoli@glide.net.in

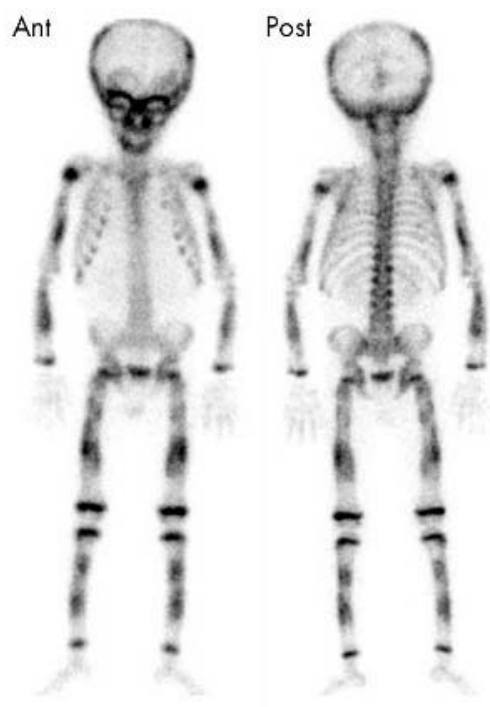

Figure 2 Whole body Tc99m MDP bone scan. 